\title{
Correlación de la productividad de un forraje con las propiedades físicas de un Ultisol en Aquidauana
}

\author{
Rafael Montanari ${ }^{1}$ Elizeu de Souza Lima², Lenon Henrique Lovera ${ }^{3}$ Luis Tadeu Rios Godoy ${ }^{3}$ \\ André Felipe Henrique ${ }^{3}$, Flávio Carlos Dalchiavon ${ }^{4}$
}

\section{RESUMEN}

Los sistemas de producción de ganadería y de manejo de pastos alteran las propiedades físicas del suelo, reflejados en la productividad y la composición vegetal. El objetivo de este trabajo fue indicar las propiedades físicas del suelo con mejores correlaciones lineales y espaciales para obtener sitios específicos de manejo del suelo. Se analizaron las correlaciones lineales y espaciales entre la productividad de la materia seca del forraje de Brachiaria decumbens (Stapf) y algunas propiedades físicas de un Ultisol en dos profundidades (0,00-0,10 m y 0,10-0,20 m). Para la colecta de los datos fue instalada una red geoestadística con 121 puntos de muestreo. La productividad del forraje varió aleatoriamente y no siguió patrones espaciales, presentando el efecto pepita puro. La resistencia mecánica del suelo a la penetración y la humedad del suelo en la capa de 0,10-0,20 m, son importantes indicadores de la calidad física del suelo. El aumento de estas propiedades en el cultivo del forraje, promovió la reducción de la materia seca del forraje. Por lo tanto, estos indicadores representan confiabilidad para estimar la productividad del forraje.

Palabras clave: resistencia mecánica del suelo a la penetración, manejo del suelo, densidad del suelo.

\section{RESUMO}

\section{Correlação entre a produtividade de forragem com atributos físicos de um Argissolo em Aquidauana}

O sistema de produção pecuária e os sistemas de manejo do pasto alteram os atributos físicos do solo, refletindo sobre a produtividade e a composição vegetal. O objetivo deste trabalho foi indicar os atributos do solo com melhores correlações lineares e espaciais para se obter zonas específicas de manejo do solo. Foram analisadas as correlações lineares e espaciais entre a produtividade de matéria seca da forragem de Brachiaria decumbens (Stapf) e de alguns atributos físicos de um Argissolo em duas profundidades (0,00-0,10 m e 0,10-0,20 m). Para isso foi instalada uma malha geoestatística para a coleta dos dados contendo 121 pontos de amostragem. A produtividade da forragem variou aleatoriamente e não seguiu padrões espaciais, sendo assim efeito pepita puro. O aumento da resistência mecânica do solo à penetração e da densidade do solo, no cultivo da forragem, causou redução na produção de matéria seca da forragem. A resistência mecânica do solo à penetração e a umidade do solo camada de 0,10-0,20 m são importantes indicadores da sua qualidade física. Portanto, representam confiabilidade para a estimativa da produtividade da forragem.

Palavras-chave: resistência mecânica do solo à penetração, manejo do solo, densidade do solo.

Recebido para publicação em 19/10/2011 e aprovado em 03/10/2012.

${ }^{1}$ Ingeniero Agrónomo, Doctor. Departamento de Fitosanidad, Ingeniería Rural y Suelos, Universidade Estadual Paulista, UNESP-FEIS, Campus de Ilha Solteira, Rua Monção, 226, 15385-000, Ilha Solteira, SP, Brasil. montanari@agr.feis.unesp.br (autor correspondiente).

${ }^{2}$ Académico de la carrera de Ingeniería Forestal. Universidade Estadual de Mato Grosso do Sul, Unidade de Aquidauana, Rodovia Aquidauana-UEMS, Km12, 79200-000, Aquidauana, MS, Brasil. elizeu_souza_lima@ hotmail.com

${ }^{3}$ Académicos de la carrera de Ingeniería Agronómica. Universidade Estadual de Mato Grosso do Sul, Unidade de Aquidauana, Rodovia Aquidauana-UEMS, Km12, 79200-000, Aquidauana, MS, Brasil. lenon_lovera@ @otmail.com; luis_godoy01@hotmail.com; andre_felipe_98@ hotmail.com;

${ }^{4}$ Ingeniero Agrónomo, Master, Estudiante de Doctorado en Sistemas de Producción, Universidade Estadual Paulista, UNESP-FEIS, Campus de Ilha Solteira, Rua Monção, 226, 15385-000, Ilha Solteira, SP, Brasil. fcdalchiavon@unemat.com 


\section{INTRODUCCIÓN}

En Brasil, la degradación de los suelos y los bajos índices de productividad de los pastos tiene merecido, por parte de la clase agronómica y de los productores rurales, especial atención. En la sabana, pastos con manejo inadecuado conducen a la degradación del suelo, llevando a limitaciones tanto por la compactación como por la pérdida de la fertilidad (Cavallini et al., 2010). La importancia de los pastos para la producción de bovinos en Brasil se debe, entre otros factores, a su bajo costo de producción (Santos et al. 2009). Por lo tanto, la recuperación de las áreas degradadas, por medio de manejos específicos, cuya implementación depende del mejor entendimiento de los mecanismos relacionados y de los límites que se deben respetar en el uso sostenible, es una de las vías para obtener aumento de la producción de carne (Páscoa y Costa, 2007).

Brasil tiene el mayor rebaño ganadero comercial del mundo, hoy día el Estado de Mato Grosso do Sul es una de las unidades federativas que más contribuye con el desarrollo agropecuario del país. En el sector pecuario, el ganado de corte brasileño es caracterizado por la explotación extensiva de los forrajes con baja productividad e índices zootécnicos insatisfactorios, comparándose a otros países que exportan carne (Cavallini et al., 2010; Pariz et al., 2011).

En la búsqueda por mejorar los índices de calidad del suelo, Larson y Pierce (1994) propusieron un conjunto importante de indicadores de orden físico, que se deberían considerar en términos de disminución de la degradación del suelo, tales como: textura, densidad del suelo y contenido de agua. La degradación de los pastos ha sido problemática para el ganado de corte, afectando directamente la sustentabilidad del sistema productivo y disminuyendo su productividad pecuaria. Se estima que cerca del $85 \%$ de los forrajes cultivados en el Centro-Oeste de Brasil, responsables por más de la mitad de la producción de carne bovina del país, se encuentran en estado de degradación. Sumado a la preocupación por los altos costos de la actividad, en los últimos años creció la atención por la degradación del suelo y sus consecuencias en la sostenibilidad de la actividad agropecuaria nacional. En áreas de cultivo de forrajes con manejo ineficiente, por lo general sin rotación y con exceso de animales, es común la presencia de capas compactadas por el pisoteo animal que causa la compactación y perdidas de suelo (Cavallini et al., 2010).

El estudio de la variabilidad de las propiedades del suelo es una etapa importante para el establecimiento de la agricultura de precisión, en la cual se pueden establecer sitios específicos de manejo (Montanari et al., 2010). En esta fase, la utilización de los semivariogramas permite planear dibujos óptimos de muestreo del suelo, con el objetivo de seleccionar la variabilidad en escalas espaciales. Una forma de solucionar estos problemas seria la adopción de métodos geoestadísticos para evaluar la variabilidad espacial de la capacidad física del suelo (Deutsch y Journel, 1998; Cavallini et al., 2010).

Teixeira Filho et al. (2010) estudiaron la productividad del forraje de braquiaria según las propiedades físicas de un Ferralsol, y observaron que la productividad de la materia seca fue baja en razón a la degradación física del suelo. Los problemas técnicos y económicos causados por la degradación de los pastos requiere, cuando posible, una reforma o recuperación de las áreas que, por el manejo inadecuado, se tornan improductivas, previniendo que el área se degrade totalmente e inviabilice la producción agropecuaria (Pariz, et al., 2011). Para que ocurra esta recuperación o mejora es necesario que se tomen decisiones técnicas y se realice un manejo integral del suelo, como el uso de abonos verdes, rotación y sucesión de cultivos. Con eso, la utilización de la herramienta geoestadística sumada a la actividad pecuaria de precisión y manejo adecuado de los pastos, puede contribuir de manera positiva para la recuperación de los pastos forrajeros en el Brasil (Cavallini et al., 2010; Pariz et al., 2011).

En el intento de establecer un indicador de la calidad física del suelo que represente las posibles causas de degradación de los forrajes de Brachiaria decumbens en Aquidauana, Estado de Mato Grosso do Sul, Brasil, y con base en datos de productividad de la masa seca del forraje (MSF), resistencia mecánica a la penetración (RP), densidad (DS), humedad gravimétrica (HG) y humedad volumétrica (HV) del suelo, este trabajo objetivó: a) analizar la variabilidad de esas propiedades, e b) definir las correlaciones lineales y espaciales entre MSF de la planta y las propiedades físicas del suelo.

\section{MATERIAL Y MÉTODOS}

El trabajo fue desarrollado en el año agrícola 2010/ 2011, en la Hacienda de Enseñanza, Investigación y Extensión de la Universidad Estadual de Mato Grosso do Sul - UEMS, ubicada en el municipio de Aquidauana, en la latitud $20^{\circ} 26^{\prime} 27^{\prime \prime S}$ y en la longitud 55³9'56"W. El clima, según la clasificación de Köppen, es del tipo tropical lluvioso de savannah, subtipo Aw, caracterizado por la distribución estacional de las lluvias, con un período seco bien definido durante los meses más fríos del año otoño/ invierno, de mayo a septiembre, y un período lluvioso durante los meses de primavera/verano, de octubre a abril.

Los datos de precipitación pluvial, humedad media relativa del aire y temperaturas máximas, medias y mínimas diarias fueron registradas a lo largo del período experimental, por la estación meteorológica de la Universidad Estadual de Mato Grosso do Sul - UEMS, Unidade de Aquidauana, ubicada a una distancia de cerca de 1.000 metros del sitio de estudio. 
La red geoestadística fue instalada en el suelo clasificado por Demattê (1980), segundo el Sistema Brasileño de Clasificación de Suelos (Embrapa, 2006), como Argissolo Vermelho-Amarelo eutrófico (Ultisol). El análisis granulométrico de la capa fina de las muestras recolectadas en el perfil fue realizado utilizando la solución de $\mathrm{NaOH}$ 0,1 N como dispersante químico y agitación en equipo de baja rotación, 12.000 rpm (Day, 1965). La arcilla fue determinada por el método de la pipeta, mientras que las fracciones de arena fueron separadas con tamiz y el limo fue calculado por diferencia. Para la profundidad de 0,00-0,10 m la concentración de arena fue de $740 \mathrm{~g} \mathrm{~kg}^{-1}, \mathrm{y}$ arcilla de $190 \mathrm{~g} \mathrm{~kg}^{-1}$; para la profundidad de $0,10-0,20 \mathrm{~m}$ se obtuvieron concentraciones de $520 \mathrm{~g} \mathrm{~kg}^{-1}$ de arcilla y de $350 \mathrm{~g} \mathrm{~kg}^{-1}$ de arena.

Inicialmente, entre julio y agosto de 2010, para la caracterización química del suelo, se recolectaron diez muestras sencillas indeformadas para una muestra compuesta que representa la red del estudio en dos profundidades (0,00-0,10 m y 0,10-0,20 m). Para eso, los puntos de recolección de las muestras sencillas fueron establecidos al azar en los surcos del cultivo anterior. Las muestras se sometieron a análisis químicos de rutina para fines de análisis de fertilidad, según Raij et al. (2001). Fueron efectuadas las siguientes determinaciones: contenido de materia orgánica (MO), pH, P, K $\mathrm{K}^{+} \mathrm{Ca}^{2+}, \mathrm{Mg}^{2+}, \mathrm{H}^{+}+\mathrm{Al}^{3+}$, suma de bases (SB), capacidad de intercambio catiónico (CIC), saturación por bases (V\%) y saturación por aluminio (m\%). Los análisis químicos fueron realizados en el laboratorio de Fertilidad de suelos del Departamento de Fitosanidad, Ingeniería Rural y Suelos, de la Universidad Estadual Paulista (UNESP), Campus de Ilha Solteira.

Las propiedades fueron evaluados, a partir de una red de estudio instalada en el forraje de Brachiaria decumbens. El área de estudio presentó los siguientes ciclos de cultivo: pasto de 1995 hasta el año agrícola 2006/2007, fríjol de 2006/2007, maíz 2007/2008, braquiaria (2008/2009) manteniéndose actualmente sin ningún tipo de manejo. El último manejo del pasto forrajero fue realizado en el año de 1995, después del gradeo y fertilización con fósforo. El forraje con manejo fue utilizado para la producción extensiva de ganado vacuno de la raza Nelore.

En la red geoestadística se definieron las direcciones $x$ y $y$ del sistema de coordenadas cartesianas, en el área de pastos de braquiaria, con auxilio del teodolito para ubicación de las estacas para apuntar la red geoestadística. La red experimental se constituyó de once transecciones de $200 \mathrm{~m}$ x $200 \mathrm{~m}$, estas fueron espaciadas cada $20 \mathrm{~m}$, con puntos de muestreo en $20 \mathrm{~m} \mathrm{x} 20 \mathrm{~m}$, conteniendo un total de 121 puntos de muestreo.

Las propiedades físicas del suelo y materia seca vegetal fueron individualmente evaluadas en los puntos del muestreo, con la planta en el centro. Se evaluaron las siguientes propiedades del suelo: densidad del suelo (DS), $\mathrm{kg} \mathrm{dm}^{-3}$; humedad gravimétrica (HG), $\mathrm{kg} \mathrm{kg}^{-1}$; humedad volumétrica $(\mathbf{H V}), \mathrm{m}^{3} \mathrm{~m}^{-3}$; y la resistencia mecánica del suelo a la penetración (RP), MPa; colectados en las profundidades de 0,00-0,10 y 0,10-0,20 m, siendo identificados en función de las profundidades de colecta: a) 0,00-0,10 m: DS1,UG1, UV1 y RP1; b) 0,10-0,20 m: DS2, UG2, UV2 y RP2.

Para la determinación de las propiedades físicas del suelo se siguieron los métodos recomendados por Embrapa (1979). Las muestras para determinación de la humedad gravimétrica fueron recolectadas directamente en los perfiles abiertos, con anillo volumétrico, para evaluar la densidad. La resistencia mecánica del suelo a la penetración se obtuvo con un penetrómetro de impacto. La expresión utilizada para el cálculo de la resistencia mecánica del suelo a la penetración fue determinada según Stolf (1991), y está presentada en la ecuación 01:

$$
\mathrm{RP}=\{5,581+6,891 \times[\mathrm{N} /(\mathrm{P}-\mathrm{A}) \times 10]\} \times 0,0981
$$

en que: RP es la resistencia mecánica del suelo a la penetración ( $\mathrm{MPa})$; $\mathbf{N}$ es el número de impactos efectuados con el martillo del penetrómetro para la obtención de la lectura; y A y $\mathbf{N}$ son las lecturas antes y después de la realización de los impactos $(\mathrm{cm})$.

La humedad volumétrica fue calculada según la ecuación 02.

HV $=H G \times D S$

en que: HV es la humedad volumétrica $\left(\mathrm{m}^{3} \mathrm{~m}^{-3}\right) ; \mathbf{H G}$ es la humedad gravimétrica $\left(\mathrm{kg} \mathrm{kg}^{-1}\right)$; y DS es la densidad del suelo $\left(\mathrm{kg} \mathrm{dm}^{-3}\right)$.

La productividad de materia verde del forraje Brachiaria decumbens se recolecto en campo y se transformó en masa de materia seca del forraje (MSF), después del secado en estufa, a $65^{\circ} \mathrm{C}$ hasta obtener un peso constante. El área útil de recolección fue de $1 \mathrm{~m}^{2}$. Para el muestreo se utilizó un cuadrado de metal con laterales de un metro, puesto en el centro del punto de muestreo. La altura del corte fue de $0,15 \mathrm{~m}$ y los resultados fueron extrapolados para $\mathrm{kg} \mathrm{ha}^{-1}$.

Los datos se sometieron a análisis estadístico descriptivo inicial, de correlación y geoestadística. Para cada variable estudiada fueron calculados la media, mediana, moda, error patrón de la media, valores de mínimo y máximo, desviaciónpatrón, varianza, coeficiente de variación, curtosis y asimetría utilizando el aplicativo computacional SAS (Schlotzhaver y Littell, 1997). Posteriormente se realizó el análisis de la distribución de frecuencia de las variables evaluadas. Para comprobar la hipótesis de normalidad y lognormalidad de los datos, fue utilizado el test de Shapiro y Wilk a $1 \%$.

La corrección de los outliers fue realizada, variable por variable, considerando exclusivamente el análisis descriptivo efectuado en SAS; esta corrección fue calculada por la media aritmética de los valores de los puntos circunvecinos en la red de estudio, sustituyendo el valor de tal outlier. El análisis de la correlación lineal sencilla 
entre todas las características estudiadas (suelo y planta) permitió seleccionar aquellos con mayor valor de " $r$ " (correlación lineal), objetivando la realización del semivariograma cruzado y consecuentemente el cokrigeado, utilizando el aplicativo computacional Excel (Montanari et al., 2010).

En cuanto al análisis geoestadístico, para cada variable, fue analizado la dependencia espacial por el cálculo del semivariograma, con base en los presupuestos de estacionalidad de la hipótesis intrínseca, con el programa Gama Design Software (Robertson, 2004). Los semivariogramas sencillos fueron ajustados a todas las variables de estudio, tanto de la planta como del suelo. Los semivariogramas ajustados en función de sus modelos, fueron efectuados por la selección inicial de: menor suma de los cuadrados de los residuos (SCR); mayor coeficiente de determinación espacial $\left(\mathrm{r}^{2}\right)$; y mayor evaluador de la dependencia espacial (EDE). La elección final del modelo que mejor representó el ajuste fue realizada por medio de la validación cruzada, bien como la definición del tamaño del "alcance" que proporcionó la mejor red de krigeado.

\section{RESULTADOS Y DISCUSIÓN}

En la tabla 1 están representados los análisis iniciales de las propiedades químicos del suelo para las profundidades de 0,00-0,10 y 0,10-0,20 m. Para las propiedades químicas estudiadas se puede observar que exclusivamente el fósforo fue el nutriente que presentó baja disponibilidad en las dos capas de suelo estudiadas $\left(9 \mathrm{mg} \mathrm{dm}^{-3}\right)$. Lo anterior, asociado probablemente a la falta de fertilizante con fósforo y también a la naturaleza ácida de los suelos brasileños que presentan gran capacidad de fijación del fósforo, indisponible para las plantas. Los valores para la caracterización inicial de la fertilidad de suelo y sus clases de evaluación según Raij et al. (1991) son presentados a seguir, respectivamente para las dos capas estudiadas: a) MO (15; $15 \mathrm{~g} \mathrm{dm}^{-3}$ - contenido medio), b) pH (5,3; 5,1 bajo), c) $\mathrm{K}^{+}\left(3,8 ; 2,7 \mathrm{mmol} \mathrm{dm}^{-3}\right.$ - contenido medio a alto), d) $\mathrm{Ca}^{2+}\left(18,7 ; 20 \mathrm{mmol}_{\mathrm{c}} \mathrm{dm}^{-3}\right.$ - contenido alto), e) $\mathrm{Mg}^{2+}(5,4$; $5,1 \mathrm{mmol} \mathrm{dm}^{-3}$ - contenido medio), f) $\mathrm{H}^{+}+\mathrm{Al}^{3+}\left(19 ; 19 \mathrm{mmol}_{\mathrm{c}}\right.$ $\mathrm{dm}^{-3}$ - contenido medio $\left.), \mathrm{g}\right) \mathrm{Al}^{3+}\left(0 ; 1 \mathrm{mmol}_{\mathrm{c}} \mathrm{dm}^{-3}-\right.$ contenido bajo), h) SB (28; $27 \mathrm{mmol}_{\mathrm{c}} \mathrm{dm}^{-3}-$ contenido medio), i) CIC (47; $48 \mathrm{mmol}_{\mathrm{c}} \mathrm{dm}^{-3}$ - contenido medio), j) V\% $(58 ; 55 \%$ - contenido medio) y k) $\mathrm{m} \%(2,2 ; 4,4 \%-$ contenido bajo). De acuerdo a los valores presentados se puede inferir que el suelo es eutrófico y químicamente adecuado para la producción del pasto Brachiaria decumbens.

Conforme Pimentel-Gomes y Garcia (2002), la variabilidad de un parámetro o variable de estudio puede ser clasificada según la magnitud de su coeficiente de variación $(\mathrm{CV})$. Sus clases fueron determinadas como baja (CV d" 10\%), media (10\% < CV d" 20\%), alta (20\% < CV d" $30 \%)$ y muy alta $(\mathrm{CV}>30 \%)$. La producción de materia seca del forraje presentó una variabilidad muy alta con un coeficiente de variación del $71 \%$ (Tabla 1). Cavallini et al. (2010) y Pariz et al. (2011) encontraron variabilidad muy alta para la producción de materia seca del forraje de braquiaria con CV's de 32,6\% y 29,7\%. La variabilidad muy alta en el presente estudio puede ser consecuencia de la baja humedad del suelo en la época del muestreo. Las muestras tanto del suelo como de la planta fueron recolectados en septiembre, mes seco en Mato Grosso do Sul. Además, el suelo estaba compactado y en esas condiciones la cubierta del forraje era heterogénea, con mucho material vegetal en algunos de los puntos de muestreo y poco en otros (Tabla 1).

Entre las propiedades estudiadas la densidad del suelo (DS) fue la única que presentó baja variabilidad en las dos capas $(4,06 \%$ y $3,55 \%)$ (Tabla 1$)$. Estos resultados son similares a los encontrados por Schaffrath et al. (2008), Faraco et al. (2008) y Cavallini et al. (2010) con variación entre 4,1-6,0\%, y en los trabajos de Montanari et al. (2010) y Pariz et al. (2011). Por lo tanto, se verifica que la densidad del suelo independientemente de la condición del cultivo, en Ferralsoles y Ultisoles, presenta baja variabilidad.

Las propiedades de resistencia mecánica del suelo a la penetración (RP), humedad gravimétrica (HG) y humedad volumétrica (HV) presentaron coeficientes de variación entre medio y alto, en un rango de 10,1-21,9\%, (Tabla 1), siendo la RP la propiedad del suelo que mostró mayor variabilidad entre las estudiadas. Es probable que esa alta variación ocurra por el pisoteo animal, como observado por Pariz et al. (2011). La humedad tanto gravimétrica como volumétrica presentó variabilidad media. Faraco et al. (2008), en el estudio de un Ferralsol, observaron valores para RP, HG y HV entre medios y altos. Cavallini et al. (2010), reporto valores bajos y altos para el mismo tipo de suelo. Tal variabilidad de RP, HG y HV en función del sistema de manejo, granulometría del suelo y tiempo de cultivo, siendo difícil la normalización del coeficiente de variación, cuando comparada con trabajos encontrados en la literatura.

El valor medio a bajo de la masa de materia seca del forraje (MSF) fue de $2963 \mathrm{~kg} \mathrm{ha}^{-1}$ (Tabla 1), considerado un promedio bajo para el forraje de braquiaria. Un promedio apreciable estaría arriba de $4000 \mathrm{~kg} \mathrm{ha}^{-1}$ (CONAB, 2011). Ese valor medio de MSF fue parecido a los $2184 \mathrm{~kg} \mathrm{ha}^{-1}$ encontrados por Pariz et al. (2011) que trabajaron con Brachiaria decumbens, pero fue distinto del obtenido por Cavallini et al. (2010) al estudiar la Brachiaria brizantha (Hochst Stapf) en una red geostadística de 124 puntos y un área de $4000 \mathrm{~m}^{2}$ en un Ferralsol. Esos últimos autores encontraron un valor promedio de $12.485 \mathrm{~kg} \mathrm{ha}^{-1}$ de masa de materia seca cuando los valores de resistencia a la penetración fueron de 2,200 y 4,557 MPa para las respectivas profundidades de $0,00-0,10$ y $0,10-0,20 \mathrm{~m}$. La evaluación de la densidad y de la resistencia mecánica del suelo a la penetración, contribuye a la determinación del 
grado de compactación, del perfil del suelo, y su efecto directo sobre el desarrollo radicular, posibilitando el establecimiento de los valores límites que restringen el crecimiento de las raíces y disminuyen la productividad.

Los promedios de la resistencia mecánica del suelo a la penetración y densidad del suelo para las profundidades de 0,00-0,10 y 0,10-0,20 m fueron respectivamente, $\mathrm{RP} 1=5,342 \mathrm{MPa}$; RP2=4,180 MPa, y DS1=1,621 kg dm ${ }^{-3}$; $\mathrm{DS} 2=1,623 \mathrm{~kg} \mathrm{dm}^{-3}$ considerados altos, indicando que el suelo estudiado se encontraba compactado según Arshad et al. (1996) y Oliveira y Moniz, (1975), que citan valores de resistencia mecánica del suelo a la penetración arriba de $2 \mathrm{MPa}$ y de densidad del suelo superior a $1,2 \mathrm{~kg} \mathrm{dm}^{-3}$ como indicadores de restricción al desarrollo de las raíces. Los valores medios para los contenidos de agua, tanto gravimétrica (HG1 $=0,080 \mathrm{~kg} \mathrm{~kg}^{-1} ; \mathrm{HG} 2=0,095 \mathrm{~kg} \mathrm{~kg}^{-1}$ ) como volumétrica (HV1 $=0,129 \mathrm{~m}^{3} \mathrm{~m}^{-3} ; \mathrm{HV} 2=0,153 \mathrm{~m}^{3} \mathrm{~m}^{-3}$ ) fueron bajos, lo que contribuyó para el incremento de la compactación. Cavallini et al. (2010) cuando estudiaron un pasto de braquiaria también encontraron promedios elevados para DS y RP y bajos para HV y HG.

La variabilidad espacial, horizontal y, o, vertical del suelo es producto de la interacción entre los factores y los procesos de su formación (Lima et al., 2007). La acción ejercida por el pastoreo animal constituye un factor decisivo para el aumento de su heterogeneidad espacial (Bertol et al., 2006). Así, tanto para la densidad (DS), cuanto para la resistencia mecánica del suelo a la penetración (RP), adicionalmente al factor de compactación del suelo por el pisoteo animal, los mayores CV's verificados en las dos profundidades, mostraron que el pastoreo a lo largo de varios años agregó heterogeneidad al área estudiada.

La productividad de la materia seca del forraje (MSF) presentó distribución no determinada, con los valores medio, máximo y mínimo de 2963, 461 y $9000 \mathrm{~kg} \mathrm{ha}^{-1}$ (Tabla 1), siendo el primero similar al promedio observado por Pariz et al. (2011) en un Ferralsol cubierto con forraje de Brachiaria decumbens en Selvíria, al de Mato Grosso do Sul. Posiblemente, el pasto en estudio se encontraba en degradación, por la pérdida del vigor del rebrote y de la capacidad natural de cubierta del suelo (abono verde). Además, muchos sitios sin presencia de forraje, con áreas expuestas al impacto de las gotas de lluvia que aumentan la compactación del suelo por el efecto del "salpico", formando capas compactadas e iniciando la erosión laminar.

Todas las propiedades físicas del suelo estudiadas en las dos profundidades presentaron distribución de frecuencia del tipo normal, así la medida de tendencia central que mejor lo representa es la media, con coeficientes de asimetría positiva entre 0,004 y 0,530 , y negativa entre $-0,446$ y $-0,223$. Esta normalidad se puede atribuir a que el suelo estaba sin manejo desde hace 15 años atrás aproximadamente, homogeneizándolo, o sea, incrementando la dependencia espacial. Los coeficientes de curtosis presentaron valores positivos entre 0,098 y 1,202 , y negativo de $-0,159$. Con todo, e independientemente de esos coeficientes, las propiedades estudiadas fueron significativas en el test de normalidad de Shapiro y Wilk al nivel de $5 \%$, dado que la probabilidad varió entre 0,101 y 0,768 (Tabla 1). De esa forma, la distribución de frecuencia del tipo normal obtenida para las propiedades físicas del suelo, así como las magnitudes de sus coeficientes de asimetría y curtosis, por lo general, en correspondieron con los datos obtenidos por Montanari et al. (2010) y Cavallini et al. (2010).

Las correlaciones entre la MSF y las propiedades del suelo (Tabla 2) fueron significativas para los pares MSF x

Tabla 1. Análisis descriptivo inicial de la productividad de la materia seca del forraje y de algunas propiedades físicas de un Ultisol, en Aquidauana, MS

\begin{tabular}{|c|c|c|c|c|c|c|c|c|c|c|}
\hline \multirow{3}{*}{ Variables ${ }^{\text {(a) }}$} & \multicolumn{10}{|c|}{ Medidas estadísticas descriptivas } \\
\hline & \multirow{2}{*}{ Media } & \multirow{2}{*}{ Mediana } & \multicolumn{2}{|c|}{ Valor } & \multirow{2}{*}{$\begin{array}{l}\text { Desvío } \\
\text { Patrón }\end{array}$} & \multicolumn{3}{|c|}{ Coeficiente } & \multicolumn{2}{|c|}{$\begin{array}{c}\text { Probabilidad } \\
\text { del test }^{(\mathbf{b})}\end{array}$} \\
\hline & & & Mínimo & Máximo & & $\begin{array}{c}\text { Variación } \\
(\%)\end{array}$ & Curtosis & Asimetría & $\operatorname{Pr}<w$ & DF \\
\hline $\operatorname{MSF}\left(\mathrm{kg} \mathrm{ha}^{-1}\right)$ & 2963,7 & 2832,5 & 461,2 & 9000 & 2100,2 & 70,9 & - & - & - & ND \\
\hline RP1 (MPa) & 5,342 & 5,279 & 2,373 & 8,524 & 1,171 & 21,9 & 0,372 & 0,345 & 0,1860 & NO \\
\hline RP2 (MPa) & 4,180 & 4,140 & 2,543 & 6,790 & 0,765 & 18,3 & 0,445 & 0,530 & 0,1007 & NO \\
\hline $\operatorname{DS} 1\left(\mathrm{~kg} \mathrm{dm}^{-3}\right)$ & 1,621 & 1,627 & 1,406 & 1,784 & 0,065 & 4,1 & 0,589 & $-0,446$ & 0,2299 & NO \\
\hline $\mathrm{DS} 2\left(\mathrm{~kg} \mathrm{dm}^{-3}\right)$ & 1,623 & 1,623 & 1,475 & 1,780 & 0,057 & 3,5 & $-0,159$ & 0,187 & 0,7287 & NO \\
\hline HG1 $\left(\mathrm{kg} \mathrm{kg}^{-1}\right)$ & 0,080 & 0,080 & 0,049 & 0,111 & 0,011 & 13,8 & 0,098 & 0,048 & 0,7680 & $\mathrm{NO}$ \\
\hline $\mathrm{HG} 2\left(\mathrm{~kg} \mathrm{~kg}^{-1}\right)$ & 0,095 & 0,095 & 0,064 & 0,130 & 0,010 & 10,9 & 0,626 & 0,004 & 0,5148 & $\mathrm{NO}$ \\
\hline $\operatorname{HV} 1\left(\mathrm{~m}^{3} \mathrm{~m}^{-3}\right)$ & 0,129 & 0,129 & 0,076 & 0,197 & 0,019 & 15,1 & 1,091 & 0,321 & 0,1884 & NO \\
\hline $\operatorname{HV} 2\left(\mathrm{~m}^{3} \mathrm{~m}^{-3}\right)$ & 0,153 & 0,154 & 0,084 & 0,211 & 0,019 & 12,6 & 1,202 & $-0,223$ & 0,2154 & $\mathrm{NO}$ \\
\hline
\end{tabular}

(a) MSF = producción de materia seca del forraje; RP, DS, HG, HV, de 1 a 2, son respectivamente la resistencia mecánica del suelo a la penetración, densidad del suelo, humedad gravimétrica y humedad volumétrica, evaluados en las capas del suelo; ${ }^{(\mathbf{b})}$ DF = distribución de frecuencia, siendo NO y ND respectivamente del tipo normal y no determinado. 
$\mathrm{RP} 2\left(\mathrm{r}=-0,196^{*}\right), \operatorname{MSF} x \mathrm{HG} 2\left(\mathrm{r}=-0,237^{* *}\right)$ y MSF x HV2 (r $\left.=-0,251^{* *}\right)$. La relación inversa (indirecta, o sea cuando aumenta el valor de una propiedad, disminuye el valor de la otra propiedad) de los tres pares presentados se evidenció que con el aumento de la resistencia mecánica del suelo a la penetración y de la humedad en la capa de 0,10-0,20 m de profundidad hubo una disminución de la MSF, producto del incremento de la compactación del suelo debido al pisoteo animal, formando una "capa de compactación en profundidad” en la capa de 0,40 m, y por lo tanto, probablemente por las condiciones adversas al crecimiento radicular, una vez que se observaron mayores concentraciones de raíces de braquiaria en las capas más profundas del suelo donde no hubo pastoreo. Cavallini et al. (2010) también encontraron correlaciones entre la MSF de braquiaria las propiedades físicas del suelo siendo estas: a) MSF x RP2 $\left(r=-0,242^{* *}\right)$ y b) MSF x HV3 ( $\left.r=-0,193^{*}\right)$.

Chaieb et al. (1996), investigando el pasto de Cenchrus cilliaris (L.), con dos a tres cortes, observaron que el sistema radicular permaneció superficial, con 58 a $67 \%$ de las raíces ubicadas en los primeros $15 \mathrm{~cm}$ de la capa superficial del suelo. Para las gramíneas Digitaria commutata (Shult) y Stipa lagascae (Roemer y Shultz) se presentó profundización de las raíces, ubicándose del 68 a $86 \%$ a $30 \mathrm{~cm}$ de profundidad. Sin embargo, cuando realizado un solo corte las tres especies se comportaron de la misma forma en comparación al tratamiento control (ausencia de corte o crecimiento libre), o sea, $85 \%$ del sistema radicular se presenta en los primeros $50 \mathrm{~cm}$ de la capa superficial del suelo y los $15 \%$ restantes entre 50 y $75 \mathrm{~cm}$. Para pastos forrajeros tropicales, Kanno et al. (1999) relataron un patrón similar de respuesta al comparar cinco gramíneas (Brachiaria decumbens cv. Basilisk, B. brizantha cv. Marandu, Panicum máximum (L.) cv. Tanzânia, P. máximum cv. Tobiatã y Andropogus gayanus (Kunth) cv. Baeti. Algunos autores concluyeron que Brachiaria brizantha es la mejor opción para ser utilizada en la rotación de cultivo-pasto en el mejoramiento de las propiedades del suelo en lo que se refiere a la cantidad y distribución de la biomasa radicular (Cavallini et al., 2010).

En el ámbito de las regresiones entre las variables MSF de la planta versus propiedades físicas del suelo para las profundidades de 0,10-0,20 m, fueron verificadas las relaciones: a) exponencial para: $\operatorname{RP} 2 \times \operatorname{MSF}\left(r=-0,196^{*}\right)$ y b) potenciales para: $\mathrm{HG} 2 \times \mathrm{MSF}\left(\mathrm{r}=-0,304^{* *}\right)$ y $\mathrm{HV} 2 \times \mathrm{MSF}(\mathrm{r}$ $\left.=-0,319^{* *}\right)$. De esa manera, la relación exponencial entre $\mathrm{RP} 2 \times$ MSF con el modelo matemático $\mathrm{RP} 2=4,325^{\mathrm{e}-2.10-}$ $5^{*} \mathrm{xMSF}\left(\mathrm{r}=-0,196^{*}\right)$, para la regresión de la HG2 en función de la MSF presentó una relación potencial con el modelo matemático: $\mathrm{HG} 2=0,128 \cdot \mathrm{MSF}^{-0,04 * *}\left(\mathrm{r}=-0,304^{* *}\right)$ y de la regresión de la HV2 en función de la MSF presentó una relación potencial con el modelo matemático: HV2 = $0,102 \cdot \mathrm{MSF}^{-0,05^{* *}}\left(\mathrm{r}=-0,319^{* *}\right)$ indicando que cuando la MSF presentó los valores de mínimo de $461 \mathrm{~kg} \mathrm{ha}^{-1}$ y de máximo de $9000 \mathrm{~kg} \mathrm{ha}^{-1}$, los valores de mínimo y máximo calculados según las ecuaciones de regresión para RP2, HG2 y HV2 fueron de (3,617 MPa y 4,285 MPa); $\left(0,090 \mathrm{~kg} \mathrm{~kg}^{-1} \mathrm{y}\right.$ $\left.0,100 \mathrm{~kg} \mathrm{~kg}^{-1}\right)$ y $\left(0,141 \mathrm{~m}^{3} \mathrm{~m}^{-3}\right.$ y $\left.0,164 \mathrm{~m}^{3} \mathrm{~m}^{-3}\right)$, indicando que el suelo estudiado está compactado.

La humedad y la resistencia mecánica del suelo a la penetración son las propiedades más utilizadas para evaluar la compactación del suelo, teniendo una estrecha correlación con el crecimiento radicular. Así, los Utisoles y Oxisoles, en condiciones naturales y con intervención antrópica mínima, presentan en la superficie $(0,00-0,30 \mathrm{~m})$ valores de densidad del suelo entre $0,98-1,13 \mathrm{~kg} \mathrm{dm}^{-3}$ (Oliveira \& Moniz, 1975) y de resistencia mecánica a la penetración en torno de 1,32 MPa (Carneiro et al., 2009). Por otro lado, Arshad et al. (1996) relataron que valores de resistencia mecánica del suelo a la penetración arriba de 2,00 MPa limitaron el desarrollo radicular de los cultivos agrícolas en general. Según Carvalho et al. (2006), cuando estos valores oscilan entre 1,29 y 2,87 MPa no restringen la productividad de granos del cultivo de fríjol. Por lo tanto, conforme Bengough et al. (2001), para realizar la correcta interpretación final de dicha resistencia, también es conveniente, por época del muestreo, recolectar

Tabla 2. Matriz de correlación lineal sencilla entre la productividad de la materia seca del forraje y de algunas propiedades físicas de un Ultisol, en Aquidauana, MS

\begin{tabular}{|c|c|c|c|c|c|c|c|c|}
\hline \multirow{2}{*}{ Variables $^{(a)}$} & \multicolumn{8}{|c|}{ Coeficiente de correlación ${ }^{(b)}$} \\
\hline & MSF & RP1 & RP2 & DS1 & DS2 & HG1 & HG2 & HV1 \\
\hline RP1 & 0,016 & - & - & - & - & - & - & - \\
\hline RP2 & $-0,196^{*}$ & $\mathbf{0 , 3 5 7 *}$ & - & - & - & - & - & - \\
\hline DS1 & $-0,047$ & $-0,039$ & $-0,070$ & - & - & - & - & - \\
\hline DS2 & $-0,077$ & $-0,141$ & $-0,079$ & 0,114 & - & - & - & - \\
\hline HG1 & $-0,156$ & $-0,388^{* *}$ & 0,074 & 0,073 & $0,225^{*}$ & - & - & - \\
\hline HG2 & $-0,237^{* *}$ & $-0,132$ & 0,029 & 0,159 & 0,122 & $0,557^{* *}$ & - & - \\
\hline HV1 & $-0,157$ & $-0,344^{* *}$ & 0,041 & $\mathbf{0 , 3 1 4} 4^{* *}$ & $0,249^{* *}$ & $0,915^{* *}$ & $0,520^{* *}$ & - \\
\hline HV2 & $-0,251^{* *}$ & $-0,189^{*}$ & $-0,015$ & $0,218^{*}$ & $0,375^{* *}$ & $0,592^{* *}$ & $0,929^{* *}$ & $0,571^{* * *}$ \\
\hline
\end{tabular}

(a) MSF, RP, DS, HG y HV, 1 y 2, son respectivamente la productividad de la materia seca del forraje, resistencia mecánica del suelo a la penetración, densidad del suelo, humedad gravimétrica y humedad volumétrica, evaluados en las capas del suelo de $0,00-0,10$ y $0,10-0,20$ m; (b) ** significativo al $1 \%$, "significativo al $5 \%$. 
datos de densidad del suelo y de humedad actual, una vez que en relación a estos la resistencia mecánica del suelo a la penetración varia de forma directa e inversamente proporcional.

Camargo \& Alleoni (1997), en un experimento sobre compactación del suelo y desarrollo de las plantas, relataron que un valor de densidad del suelo de $1,55 \mathrm{~kg}$ $\mathrm{dm}^{-3}$ puede limitar el desarrollo vegetativo. Tal valor, así como su influencia sobre el desarrollo de los cultivos, además de estar íntimamente asociado a las características intrínsecas del suelo, tales como la textura y la estructura, también es dependiente del cultivo y del manejo del suelo (Montanari et al., 2010). El valor medio de la materia seca del forraje (MSF) fue de $2963 \mathrm{~kg} \mathrm{ha}^{-1}$ (Tabla 1), considerado como una media baja del forraje de braquiaria, puesto que el suelo se encontraba compactado y con baja humedad, siendo susceptible a degradación. Cavallini et al. (2010) logró obtener un promedio de productividad del forraje de $12.485 \mathrm{~kg} \mathrm{ha}^{-1}$ con una humedad media de $0,320 \mathrm{~m}^{3} \mathrm{~m}^{-3}$.

En la tabla 3 y la Figura 1 son presentados los parámetros de los semivariogramas sencillos ajustados para algunas de las propiedades físicas de un Ultisol de Aquidauana (MS). Se comprobó que, con excepción de MSF, RP2, DS1, HV1 y HV2, que presentaron efecto pepita puro, todos los demás presentaron dependencia espacial.

Para la HG1 y RP1, los valores de los $\mathrm{r}^{2}$ determinados fueron de 0,778 y 0,743 , los que indicaron mejor ajuste semivariográfico de las propiedades del suelo evaluadas. Estos resultados difieren de los encontrados en un Ferralsol por Rosa Filho et al. (2009), Cavallini et al. (2010) y Montanari et al. (2010) (0,816; 0,968 y 0,976 respectivamente). En relación al EDE, los valores constatados (86,0 y 89,9\%) también difirieron de los de Rosa Filho et al. (2009), los cuales fueron considerados moderados con valores de 70,0\%. Los modelos exponenciales ajustados para la HG1 y RP1 coinciden con el encontrado por Rosa Filho et al. (2009); también el alcance de la HG1 del presente trabajo (53,1 m) fue similar al de Cavallini et al. (2010) en una red de 100 x $400 \mathrm{~m}(52,7 \mathrm{~m})$, pero diferente de los datos de Pariz et al. (2011) que estudiaron la humedad en una red de 160 x $160 \mathrm{~m}$ y encontraron un alcance de $26,5 \mathrm{~m}$ (Tabla 3 y Figura 1a,c). El alcance para la RP1 del presente estudio (37,8 m) no fue observada espacialmente por Pariz et al. (2011) que encontraron el efecto pepita para la misma variable en la respectiva capa del suelo de 0,00-0,10 m. Por lo tanto, exclusivamente con base en la presente investigación, así como, buscando auxiliar a las investigaciones futuras, en el cual las mismas variables estén involucradas, los valores de los alcances de la dependencia espacial utilizados en los programas geoestadísticos, que alimentarán los programas de los ordenadores empleados en la agricultura de precisión, en general, no deberán ser menores que 37,8 m para las propiedades del suelo.

En la comparación inicial de los mapas de krigeado de las propiedades del suelo (RP1, DS2, HG1 y HG2) (Figura 2), se observa que la región Este de los mapas de RP1 y DS2 (Figuras 2a, b) son los sitios con menor resistencia mecánica del suelo a la penetración y densidad del suelo, es decir, en el caso que se usara un gradeo en el área para mejorar las condiciones físicas del mismo se utilizarían los mapas de RP1 y DS2, dado que las líneas de los mapas son georreferenciables, por lo tanto se puede para años siguientes de cultivo en la misma área establecer sitios específicos de manejo para incremento de la productividad. Para HG1 y HG2 (Figuras 2c, d) la región Sur del mapa indica los sitios de mayor contenido de agua en el suelo, ya que la parte inferior del área de estudio sentido próximo a una microbarcia. (Figura 2)

Tabla 3. Parámetros de los semivariogramas ajustados para la productividad de la materia seca del forraje y de algunas propiedades físicas de un Ultisol, en Aquidauana, MS

\begin{tabular}{|c|c|c|c|c|c|c|c|c|}
\hline \multirow{3}{*}{ Variables (a) } & \multirow{3}{*}{ Modelo $^{(\mathbf{b})}$} & \multicolumn{7}{|c|}{ Parámetros } \\
\hline & & \multirow{2}{*}{$\begin{array}{c}\text { Efecto } \\
\text { Pepita } \\
\left(\mathbf{C}_{0}\right)\end{array}$} & \multirow{2}{*}{$\begin{array}{c}\text { Meseta } \\
\left(\mathrm{C}_{0}+\mathrm{C}\right)\end{array}$} & \multirow{2}{*}{$\begin{array}{c}\text { Alcance } \\
\left(\mathbf{A}_{0}\right) \\
(\mathbf{m})\end{array}$} & \multirow[t]{2}{*}{$\mathbf{r}^{2}$} & \multirow[t]{2}{*}{$\mathbf{S C R}^{(\mathrm{c})}$} & \multicolumn{2}{|c|}{$\begin{array}{c}\text { Evaluador de la } \\
\text { dependencia espacial }\end{array}$} \\
\hline & & & & & & & $\mathbf{E D E}^{(\mathrm{d})}$ & Clase \\
\hline \multicolumn{9}{|c|}{ X(h) sencillos de las variables de la planta y del suelo } \\
\hline MSF (kg ha $\left.{ }^{-1}\right)$ & epp. & $1,640.10^{6}$ & $1,640.10^{6}$ & - & - & - & - & - \\
\hline RP1 (MPa) & exp. & $9,500 \cdot 10^{-2}$ & $9,370.10^{-1}$ & 37,8 & 0,743 & $8,769 \cdot 10^{-3}$ & 89,9 & fuerte \\
\hline RP2 (MPa) & epp . & $5,835.10^{-1}$ & $5,835.10^{-1}$ & - & - & - & - & - \\
\hline DS1 $\left(\mathrm{kg} \mathrm{dm}^{-3}\right)$ & epp. & $4,275 \cdot 10^{-3}$ & $4,275 \cdot 10^{-3}$ & - & - & - & - & - \\
\hline DS2 $\left(\mathrm{kg} \mathrm{dm}^{-3}\right)$ & exp. & $3,700 \cdot 10^{-4}$ & $2,700 \cdot 10^{-2}$ & 54,6 & 0,674 & $2,291 \cdot 10^{-7}$ & 86,3 & fuerte \\
\hline HG1 $\left(\mathrm{kg} \mathrm{kg}^{-1}\right)$ & exp. & $1,700 \cdot 10^{-5}$ & $1,170.10^{-4}$ & 53,1 & 0,778 & $2,180 \cdot 10^{-10}$ & 86,0 & fuerte \\
\hline HG2 $\left(\mathrm{kg} \mathrm{kg}^{-1}\right)$ & exp. & $5,000 \cdot 10^{-6}$ & $9,300 \cdot 10^{-5}$ & 72,3 & 0,660 & $2,762.10^{-10}$ & 94,2 & fuerte \\
\hline HV1 $\left(\mathbf{m}^{3} \mathbf{m}^{-3}\right)$ & epp. & $3,460.10^{-4}$ & $3,460.10^{-4}$ & - & - & - & - & - \\
\hline HV2 $\left(\mathbf{m}^{3} \mathbf{m}^{-3}\right)$ & epp. & $3,490 \cdot 10^{-4}$ & $3,490 \cdot 10^{-4}$ & - & - & - & - & - \\
\hline
\end{tabular}



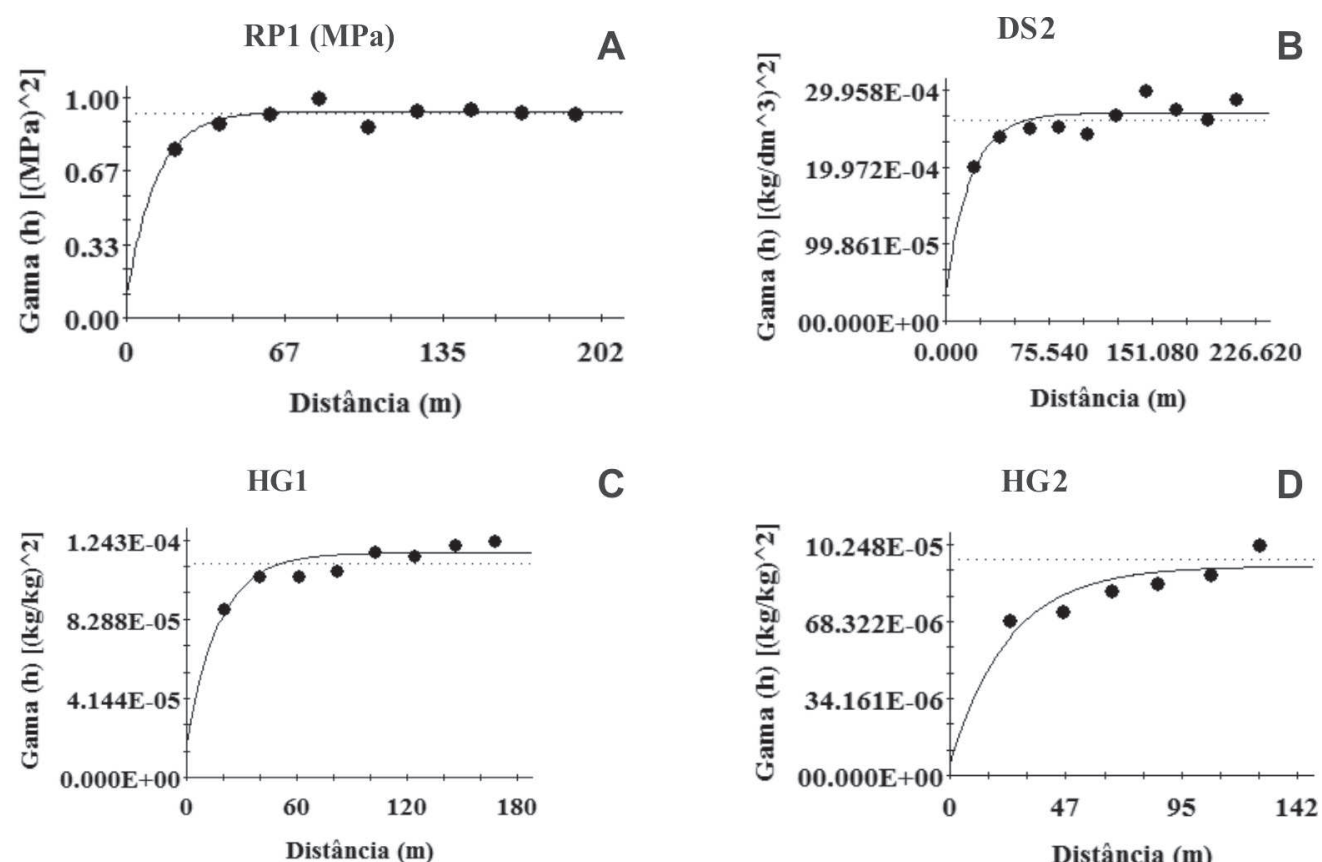

C

HG2

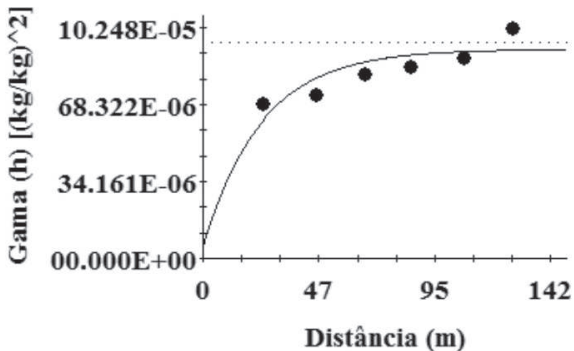

Figura 1. Semivariogramas sencillos de algunas propiedades físicas de un Ultisol, en Aquidauana, MS

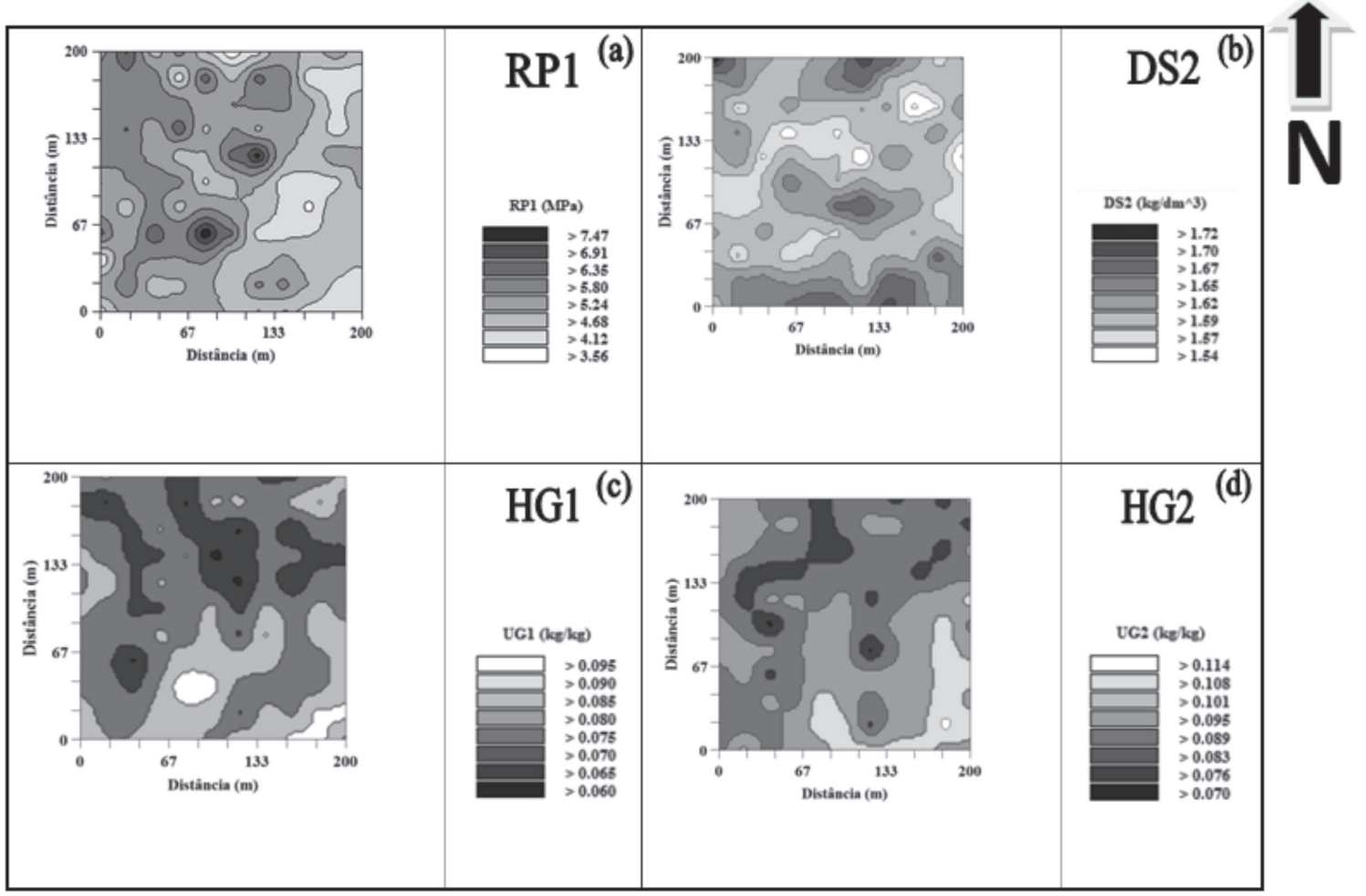

Figura 2. Mapas de krigeado de algunas propiedades físicas de un Ultisol, en Aquidauana, MS

\section{CONCLUSIONES}

La productividad de Brachiaria decumbens variaaleatoriamente y no sigue patrones espaciales bien definidos, presentando alta variabilidad, o sea, efecto pepita puro;
La resistencia mecánica a la penetración, el contenido de agua y la densidad del suelo presentaron variabilidad espacial para el área de estudio;

En este estudio la resistencia mecánica a la penetración, el contenido de agua y la densidad fueron indicadores de la calidad física del suelo. 


\section{AGRADECIMIENTOS}

$\mathrm{Al} \mathrm{CNPq}$ (PIBIC-AAF-UEMS) por la Beca de iniciación científica concedida al segundo autor.

\section{REFERENCIAS}

Arshad MA, Lower B \& Grossman B (1996) Physical tests for monitoring soil quality. In: Doran JW \& Jones AJ (Eds.) Methods for assessing soil quality. Madison, Soil Science Society of America. p.123-141 (Special Publication, 49).

Bengough AG, Campbell DJ \& O' Sullivan MF (2001) Penetrometer techniques in relation to soil compaction and root growth. In: Smith KA \& Mullins CE (Eds.) Soil and environmental analysis: physical methods. $2^{\mathrm{a}}$ ed. New York, Marcel Dekker. p.377-403.

Bertol I, Mafra AL \& Cogo NP (2006) Conservação do solo em pastagens. In: $23^{\circ}$ Simpósio sobre manejo da pastagem, Piracicaba. Anais, FEALQ. p.139-163.

Camargo AO \& Alleoni LRF (1997) Compactação do solo e desenvolvimento das plantas. Piracicaba, ESALQ. 132p.

Carneiro MAC, Souza ED, Reis EF, Pereira HS \& Azevedo WR (2009) Atributos físicos, químicos e biológicos de solo de cerrado sob diferentes sistemas de uso e manejo. Revista Brasileira de Ciência do Solo, 33:147-157.

Carvalho GJ, Carvalho MP, Freddi OS \& Martins MV (2006) Correlação da produtividade do feijão com a resistência à penetração do solo sob plantio direto. Revista Brasileira de Engenharia Agrícola e Ambiental, 10:765-771.

Cavallini MR, Andreotti M, Oliveira LL, Pariz CM \& Carvalho MP (2010) Relações entre produtividade de Brachiaria brizantha e atributos físicos de um Latossolo do Cerrado. Revista Brasileira de Ciência do Solo, 34:1007-1015.

Chaieb M, Belgacem H \& Makki B (1996) Impact of clipping on root systems of 3 grasses species in Tunisia. Journal of Range Management, 49:336-339.

Companhia Nacional de Abastecimento - CONAB (2011) Consolidado e acompanhamento da safra 2010/2011: $1^{\circ}$ levantamento. Disponível em: <www.conab.gov.br>. Acessado em: 24 de julho 2011.

Day PR (1965) Particle fractionation and particle-size analysis. In: Black CA (Ed.) Methods of soil analysis. Madison, Wisconsin. p.545-567.

Demattê JLI (1980) Levantamento detalhado de solos do "Campus Experimental de Ilha Solteira". Piracicaba, Escola Superior de Agricultura "Luiz de Queiroz". 44p.

Deutsch CV \& Journel AG (1998) GSLIB: geostatistical software library and user's guide. New York, Oxford University. 369p. (CD-ROM)

Empresa Brasileira de Pesquisa Agropecuária - Embrapa (1979) Manual de métodos de análise de solos. Rio de Janeiro, Ministério da Agricultura. 212p.

Empresa Brasileira de Pesquisa Agropecuária - Embrapa (2006) Sistema brasileiro de classificação de solos. $2^{\text {a }}$ ed. Rio de Janeiro, Embrapa. 306p.

Faraco MA, Uribe-Opazo MA, Da Silva EAA, Johann JA \& Borssoi JA (2008) Seleção de modelos de variabilidade espacial para elaboração de mapas temáticos de atributos físicos do solo e produtividade da soja. Revista Brasileira de Ciência do Solo, 32:463-476.

Robertson GP (2004) GS+: Geostatistics for environmental sciences. Version 9. Plainwell, Gamma Desing Software. 169p.
Kanno T, Macedo MCM, Euclides VPB, Bonno JA, Santos Junior JDG, Rocha MC \& Beretta LGR (1999) Root biomass of five tropical Grass pastures under continuous grazing in Brazilian Savannas. Grassland Science, 45:09-14.

Larson WE \& Pierce FJ (1994) The dynamics of soil quality as a measure of sustainable management. In: Doran JW, Coleman DC, Bezdicek DF \& Stewart BA (Eds.) Defining Soil Quality for a Sustainable Environment. Madison, Soil Science Society of America. p.37-51. (Special Publication, 35).

Lima CGR, Carvalho MP, Mello LMM \& Lima RC (2007) Correlação linear e espacial entre a produtividade de forragem, a porosidade total e densidade do solo de Pereira Barreto (SP). Revista Brasileira de Ciência do Solo, 31:1233-1244.

Montanari R, Carvalho MP, Andreotti M, Dalchiavon FC, Lovera LH \& Honorato MAO (2010) Aspectos da produtividade do feijão correlacionados com atributos físicos do solo sob elevado nível tecnológico de manejo. Revista Brasileira de Ciência do Solo, 34:1811-1822.

Oliveira JB \& Moniz AC (1975) Levantamento pedológico detalhado da Estação Experimental de Ribeirão Preto. Bragantia, 34:59-114.

Pariz CM, Carvalho MP, Chioderoli CA, Nakayama FT, Andreotti M \& Montanari R (2011) Spatial variability of forage yield and soil physical attributes of a Brachiaria decumbens pasture in the Brazilian Cerrado. Revista Brasileira de Zootecnia, 33:722729.

Páscoa AG \& Costa MJRP (2007) Aplicação dos sistemas de informação geográfica para definição de estratégias de manejo de bovinos nas pastagens. Revista Brasileira de Zootecnia, 36:4551 .

Pimentel-Gomes FP \& Garcia CH (2002) Estatística aplicada a experimentos agronômicos e florestais. Piracicaba, FEALQ. 309 p.

Raij, B van. Fertilidade do solo e adubação. Piracicaba: Ceres, 1991. 343p.

Raij B van, Andrade JC, Cantarela H, Quaggio JA (2001) Análise química para avaliação da fertilidade de solos tropicais. Campinas, Instituto Agronômico. 285p.

Rosa Filho G, Carvalho MP, Andreotti M, Montanari R, Binotti FFS \& Gioia MT (2009) Variabilidade da produtividade da soja em função de atributos físicos de um latossolo vermelho distroférrico sob plantio direto. Revista Brasileira de Ciência do Solo, 33:283-293.

Santos MER, Fonseca DM \& Euclides VPB (2009) Produção de bovinos em pastagens de capim braquiária diferidas. Revista Brasileira de Zootecnia, 38:635-642.

Schaffrath VR, Tormena CA, Fidalski J \& Gonçalves ACA (2008) Variabilidade e correlação espacial de propriedades físicas de solo sob plantio direto e preparo convencional. Revista Brasileira de Ciência do Solo, 32:1369-1377.

Schlotzhaver SD \& Littell RC (1997) SAS system for elementary statistical analysis. $2^{\mathrm{a}}$ ed. Cary, SAS. 441p.

Stolf R (1991) Teoria e teste experimental de fórmulas de transformação dos dados de penetrômetro de impacto em resistência do solo. Revista Brasileira de Ciência do Solo, 15:229-235.

Teixeira Filho MCM, Buzetti S, Andreotti M, Arf O \& Benett CGS (2010) Doses, fontes e épocas de aplicação de nitrogênio em trigo irrigado em plantio direto. Pesquisa Agropecuária Brasileira, 45:797-804.

Rev. Ceres, Viçosa, v. 60, n.1, p. 102-110, jan/fev, 2013 